\title{
In vivo study of dendronlike nanoparticles for stem cells "tune-up": from nano to tissues
}

\author{
Joaquim M. Oliveira, $\mathrm{PhD}^{\mathrm{a}, \mathrm{b}, \mathrm{c}}$, Rui A. Sousa, $\mathrm{PhD}^{\mathrm{a}, \mathrm{b}}$, Patricia B. Malafaya, $\mathrm{PhD}^{\mathrm{a}, \mathrm{b}}$, \\ Simone S. Silva, $\mathrm{PhD}^{\mathrm{a}, \mathrm{b}}$, Noriko Kotobuki, $\mathrm{PhD}^{\mathrm{c}}$, Motohiro Hirose, $\mathrm{PhD}^{\mathrm{c}}$, \\ Hajime Ohgushi, MD, $\mathrm{PhD}^{\mathrm{c}}$, João F. Mano, $\mathrm{PhD}^{\mathrm{a}, \mathrm{b}}$, Rui L. Reis, $\mathrm{PhD}^{\mathrm{a}, \mathrm{b}, *}$ \\ ${ }^{a}$ Biomaterials, Biodegradables and Biomimetics Research Group, Department of Polymer Engineering, University of Minho, Headquarters of the European \\ Institute of Excellence on Tissue Engineering and Regenerative Medicine, Guimarães, Portugal \\ ${ }^{\mathrm{b}}$ Institute for Biotechnology and Bioengineering, PT Government Associated Laboratory, Braga, Portugal \\ ${ }^{\mathrm{c}}$ Research Institute for Cell Engineering (RICE), National Institute of Advanced Industrial Science and Technology (AIST), Amagasaki, Japan
}

Received 3 September 2010; accepted 5 March 2011

\begin{abstract}
The control of stem cell differentiation to obtain osteoblasts in vivo is still regarded as a challenge in stem-cell-based and bone-tissue engineering strategies. Biodegradable dexamethasone-loaded dendron-like nanoparticles (NPs) of carboxymethylchitosan/poly(amidoamine) dendrimer have been proposed as intracellular drug-delivery systems of bioactive molecules. In this study, combination of nanotechnology, stem-cell engineering and tissue engineering is proposed in pre-programming the fate of rat bone marrow stromal cells (RBMSCs) towards osteoblasts cells and development of new bone tissue, in vivo. This work demonstrated that the developed NPs were able to be taken up by RBMSCs, and exhibited a noncytotoxic behavior in vitro. The performance of the developed dendronlike NP system for the intracellular delivery of dexamethasone was investigated by seeding the engineered RBMSCs onto starch-polycaprolactone scaffolds ex vivo, and implanting subcutaneously in the back of Fischer 344/N rats (Syngeneic), in the absence of the typical osteogenic supplements. Favorable results were observed in vivo, thus suggesting that stem cell "tune-up" strategy can open up a new regenerative strategy for bone-tissue engineering.
\end{abstract}

From the Clinical Editor: In this study, a combination of nanotechnology, stem-cell engineering and tissue engineering is proposed in preprogramming the fate of rat bone marrow stromal cells (RBMSCs) towards osteoblasts cells and development of new bone tissue in vivo. (C) 2011 Elsevier Inc. All rights reserved.

Key words: Bone; Cytotoxicity; Internalization; In vivo; Nanoparticles; Stem cells

Advanced regenerative therapies comprise the fields of stemcell engineering, tissue engineering and gene therapy. Cell engineering and tissue engineering have benefited from the

The authors would like to thank the financial support from Portuguese Foundation for Science and Technology (FCT, project SmartCarbo, ref. PTDC/QUI/68804/2006), through POCTI and FEDER programs. The funding provided by Canon Foundation in Europe is gratefully acknowledged. This work was also carried out under the scope of the European NoE EXPERTISSUES (NMP3-CT-2004-500283) and HIPPOCRATES (NMP3CT-2003-505758) projects.

*Corresponding author: 3B's Research Group - Biomaterials, Biodegradables and Biomimetics, Department of Polymer Engineering, University of Minho, Headquarters of the European Institute of Excellence on Tissue Engineering and Regenerative Medicine, Taipas 4806-909, Guimarães, Portugal.

E-mail address: rgreis@dep.uminho.pt (R.L. Reis). development of novel strategies ${ }^{1-3}$ that can stimulate and control cells' functions in vitro and in vivo. This issue is still regarded as a very appealing challenge ${ }^{4-6}$ because there is the need to targetdeliver biological agents, including differentiation factors or genetic material towards modulating from inside stem cells' behavior. We find it interesting that You et $\mathrm{al}^{7}$ reported that surface nanotopography of polyurethane polymer functionalized with acrylate groups can enhance osteogenic differentiation synergistically with biochemical induction substance in vitro. Nevertheless, nanocarrier systems have generated a significant amount of interest in ex vivo cell maintenance and co-culturing, ${ }^{8,9}$ and for tuning the cellular fate in vivo mainly due to their internalization ability and drug-loading capacity and to favorably modulate the solubility and pharmacokinetics of drugs. ${ }^{10,11}$ Likewise, much attention has been given ${ }^{12,13}$ to understanding the fundamental problem of the entry and retention mechanisms of nanoparticles (NPs) on mammalian cells as the nanodevices may be incorporated into membrane- 
bounded endosomes and fail to access the cytosolic cell targets. In this regard dendrimers have attracted growing interest as nanocarriers due to their ability to cross cell membranes. ${ }^{14}$ Despite the interesting architecture and multivalency, it has been found ${ }^{15}$ that high generation amine-terminated dendrimers are often cytotoxic. Many researchers have proposed the designing of dendrimers for biological and medical applications. ${ }^{16,17}$ Dendrimer-based NPs may be biocompatible, possess high loading capacity and allow the bulk incorporation of bioactive molecules of higher molecular weights and of different chemistry while maintaining internalization and transfection efficiencies in comparison with conventional dendrimers. Still, these have not been successfully developed. We focused our attention on this fundamental problem and propose a novel surface-engineering strategy by using carboxymethylchitosan (CMCht), a natural amphoteric polyelectrolyte derived from chitosan, grafted to a low generation poly(amidoamine) dendrimer (PAMAM) backbone, with the aim of obtaining copolymers of new architectures and physicochemical properties, ${ }^{18}$ the so-called CMCht/PAMAM dendrimer NPs. This class of nanobiomaterials has been projected to act intracellularly towards tuning in vivo the stem cells' fate. However, this remains 'proof-of-concept' with a previous report ${ }^{18}$ showing that the surface engineering of PAMAM dendrimer NPs with the biodegradable CMCht possibly to manipulate the chemical composition and improve biocompatibility, thus avoiding the cytotoxic effects of high-generation dendrimers. Moreover, upon loading of dexamethasone (Dex), the dendrimer nanocarriers promoted the osteogenic differentiation of rat bone marrow stromal cells (RBMSCs) in vitro. ${ }^{18,19}$ The delivery of Dex by means of using the NPs possibly a intracellular and regimented supply to stem cells within the required range of concentrations, which can be advantageous for proper osteogenic differentiation.

Here, we report on the effect of Dex-loading on the architecture, zeta potential and internalization ability of the developed NPs by RBMSCs. Transmission electron microscope (TEM), dynamic light scattering (DLS) and fluorescence techniques were carried out. To further investigate their osteogenic potential, in vitro and in vivo studies were performed combining cell and tissue engineering principles, i.e., using natural nano- and micro-polymeric systems. In vitro mineralization was evaluated by measuring the levels of calcein uptake. For the in vivo studies, Dex-loaded CMCht/PAMAM dendrimer NPs were exposed from culture media to RBMSCs during the expansion period and seeded onto the surface of starchpolycaprolactone (SPCL) scaffolds, followed by subcutaneous implantation in the back of Fischer $344(\mathrm{~F} 344 / \mathrm{N})$ rats for 4 weeks. Bone formation was evaluated by microcomputed tomography (micro-CT) and morphometric analyses and histological studies (Haematoxylin \& Eosin staining). Biochemical analyses denoting osteogenesis such as ALP activity, osteocalcin content and calcium assay were also carried out.

\section{Methods}

\section{Synthesis and characterization of the NPS}

Synthesis of CMCht/PAMAM dendrimer NPs was performed in a stepwise manner as described elsewhere. ${ }^{18} \mathrm{CMCht}$ with a degree of deacetilation of $80 \%$ and degree of substitution of $47 \%$ was linked to poly(amidoamine) dendrimers with an ethylenediamine core. First, the amine-terminated poly(amidoamine) dendrimers (PAMAM-AT) was obtained from commercial Starburst carboxylic-terminated poly(amidoamine) dendrimer (PAMAM-CT, G1.5) (Aldrich, St. Louis, Missouri), as follows: (1) an appropriate volume of PAMAM-CT was dissolved in ultrapure water to give a final concentration of $10 \mathrm{mg} \cdot \mathrm{ml}^{-1}$, and the $\mathrm{pH}$ of the solution adjusted to 6.5 with dilute hydrochloride acid (HCl) (Riedel de-Haen, Seelze, Germany), (2) 1-ethyl-3(3dimethylaminopropyl) carbodiimide hydrochloride, EDC (Fluka, Buchs, Switzerland) was added to the PAMAM-CT solution at a molar ratio sufficient to modify the carboxylate residue of the dendrimers under agitation, (3) ethylenediamine (EDA) (Sigma, Steinheim, Germany) was added to the solution at a molar ratio equal to that of EDC, and (4) PAMAM-AT was produced by removing excess of EDC by dialysis using a dialysis tubing benzoylated (Sigma, Steinheim, Germany). Then, a PAMAM-ester terminated dendrimer was obtained from PAMAM-AT compound by adding an appropriate volume of PAMAM-AT ( $\sim 8.4 \mathrm{mmol})$ and $1.14 \mathrm{ml}$ of methyl methacrylate $(\sim 12.6 \mathrm{mmol})$ (Fluka) in methanol (Sigma) under agitation. The PAMAM-ester terminated compound was obtained by precipitation after 24 hours of reaction at $40^{\circ} \mathrm{C}$. At last, CMCht/ PAMAM dendrimer was obtained by removing the acetal to PAMAM-ester terminated compound ${ }^{20}$ and by adding the CMCht at a ratio of 1:2 (w:w). The final solution was diluted by adding $30 \mathrm{ml}$ of methanol and kept under agitation for 1 hour. After that time period, Dex-loaded CMCht/PAMAM dendrimer NPs were obtained via a precipitation route by mixing the CMCht/PAMAM dendrimer NPs with Dex at a final concentration of $5 \times 10^{-5} \mathrm{M}$ under agitation. NPs were obtained after precipitation by adding an appropriate volume of a saturated sodium carbonate, $\mathrm{Na}_{2} \mathrm{CO}_{3}$ (Aldrich) solution and cold acetone (Pronalab, Lisbon, Portugal). The precipitate was collected by filtration and dispersed in ultrapure water. The solution was dialyzed against deionized water for several days by means of using a dialysis tubing benzoylated (Sigma, St. Louis, Missouri). The NP powders were obtained after freezing the solution at $-80^{\circ} \mathrm{C}$ and freeze-drying (Telstar-Cryodos -80 , Terrassa, Spain) up to 4 days to completely remove the solvent. Fluorescent probe NPs were also synthesized using fluorescein isothiocyanate (FITC). ${ }^{18}$ The incorporation of Dex into the CMCht/PAMAM dendrimer NPs was investigated by ${ }^{1} \mathrm{H}$ NMR spectroscopy. CMCht/PAMAM dendrimer NPs and Dex-loaded CMCht/ PAMAM dendrimer NPs were dissolved in deuterated water, $\mathrm{D}_{2} \mathrm{O}$ (Aldrich). The NMR spectra were obtained with a Mercury - 400BB operating at a frequency of $399.9 \mathrm{MHz}$ at $50^{\circ} \mathrm{C}$. The one-dimensional ${ }^{1} \mathrm{H}$ NMR spectra were acquired using a $45^{\circ}$ pulse, a spectral width of $6.3 \mathrm{kHz}$ and an acquisition time of 2.001 seconds. The changes on the morphology of the CMCht/ PAMAM dendrimer NPs after incorporation of Dex was investigated under TEM (Philips CM-12, FEI Company, Eindhoven, The Netherlands) equipped with a MEGA VIEW-II DOCU camera and Image Software Analyzer SIS NT DOCU, as previously described elsewhere. ${ }^{18}$ In addition, zeta potential and particle-size distribution of the NPs loaded and nonloaded with Dex were investigated at physiological and lysosomal typical 
pH's using a particle-size analyzer (Zetasizer Nano ZS, Malvern Instruments, Worcestershire, United Kingdom). For this study, particle-size analyses were also performed by DLS in an aqueous solution with low concentration of NPs and using disposable sizing cuvettes. Electrophoretic determinations of zeta potential were investigated in citrate buffer $(\mathrm{pH} 3)$ and phosphate buffered saline (PBS) of $\mathrm{pH} 7.4$ solutions, using the universal 'dip' cell. Zeta potential was also investigated in water. For further details see Supplementary Information.

\section{SPCL scaffolds}

A blend of starch and poly- $\varepsilon$-caprolactone from Novamont (Novara, Italy) 30/70 (w/w) was used to produce fibers of SPCL by melt spinning using a modular co-rotating twin screw extruder. SPCL scaffolds with $5 \mathrm{~mm}$ diameter and $4 \mathrm{~mm}$ height were obtained by fiber bonding as reported elsewhere. ${ }^{19,21}$ Before in vitro and in vivo studies, the CMCht/PAMAM dendrimer NPs, the Dex-loaded CMCht/PAMAM dendrimer NPs and SPCL scaffolds were sterilized in an ethylene-oxide gas atmosphere.

\section{Cells isolation and luminescent cell viability assay (ATP quantification)}

For the in vitro cell culturing studies, RBMSCs were isolated from femora of 7-week-old male Fischer 344/N rats (SLC Inc., Hammamatsu, Japan), and expanded in $T 75 \mathrm{~cm}^{2}$ culture flasks in the presence of Eagle's minimum essential medium (MEM) (Nacalai Tesque, Kyoto, Japan) supplemented with 15\% fetal bovine serum (FBS), (JRH Biosciences, Tokyo, Japan) and 1\% antibiotic/antimycotic (A/B) (Nacalai Tesque) solution, the socalled MEM complete medium. First, the animals were sacrificed following the protocol approved by Ethics Committee at the Tissue Engineering Research Center, Amagasaki, Japan. The femora were collected, the marrow flushed out and marrow cells from each shaft were transferred into a $T 75 \mathrm{~cm}^{2}$ culture flask (BD Biosciences Discovery Labware, Bedford, Massachusetts) and expanded under standard culturing conditions. The culture medium was changed within a 3-day period to remove nonadherent cells, followed by changing the culture medium every 2 or 3 days until reaching about $80 \%$ confluence. RBMSCs were trypsinized with $1 \mathrm{ml}$ of $0.05 \%$ tripsin-0.53 mM EDTA (Invitrogen, Carlsbad, California) solution, and incubated for 3 minutes at $37^{\circ} \mathrm{C}$. Complete MEM culture medium was added and RBMSCs (passage 1, P1) were centrifuged at $900 \mathrm{rpm}$ for 5 minutes. Then, supernatant was aspirated and cells resuspended with $10 \mathrm{ml}$ of complete culture medium. Cell counting was performed using an automated counter (Cell Counter Sysmex F-520, Kobe, Japan). Viability of RBMSCs was analyzed with a NucleoCounter (Chemometec, Allerød, Denmark), prior seeding.

To investigate possible cytotoxicity of the NPs, a cellviability assay was carried out. RBMSCs were seeded (subcultured) to each well of a 96-well tissue culture polystyrene (TCPS) plate at a cell density of $5 \times 10^{3}$ cells.mll ${ }^{-1}$. Serial dilutions $\left(1,0.1,0.01 \mathrm{mg} . \mathrm{ml}^{-1}\right)$ of CMCht/PAMAM dendrimer NPs and Dex-loaded CMCht/PAMAM dendrimer NPs were prepared using the MEM complete culture medium, and RBMSCs cultured with the respective media for 24 and 72 hours. A latex rubber extract was used as the positive control for cellular death. After each time point, the ATP content was measured by means of performing a CellTiter-Glo luminescent cell-viability assay (Promega Corp., Madison, Wisconsin). Luminescence was measured in a microplate reader (Wallac ARVOsx 1420, Perkin-Elmer Life and Analytical Sciences, Shelton, Connecticut). All experiments were carried out in triplicate using 4 replicates per experimental condition $(n=12)$.

\section{In vitro cellular uptake ability of the dendronlike NPS}

RBMSCs were isolated and expanded as aforementioned. Then, cells were subcultured in a 6 -well $\left(1 \times 10^{5}\right.$ cells.well $\left.^{-1}\right)$ and TCPS coverslips in 24-well TCPS plates $\left(2 \times 10^{4}\right.$ cells.well $\left.^{-1}\right)$ for analysis under FACS and fluorescence microscopy, respectively. For fluorescence microscopy, RBMSCs were cultured in a MEM complete culture medium supplemented with the FITClabelled Dex-loaded CMCht/PAMAM dendrimer NPs and MEM complete culture medium with the FITC-labelled CMCht/PAMAM dendrimer NPs at a final concentration of $0.1 \mathrm{mg} \cdot \mathrm{ml}^{-1}$, for the period of 12 hours for 14 days. For FACS analysis, RBMSCs were cultured in the presence of FITClabelled Dex-loaded CMCht/PAMAM dendrimer NPs and FITC-labelled CMCht/PAMAM dendrimer NPs at a final concentration of $0.01 \mathrm{mg} \cdot \mathrm{ml}^{-1}$. All experiments were carried out in triplicate. After each time period, the RBMSCs were fixed with 4\% formalin (Nacalai Tesque) and incubated with Texas Red-X phalloidin (Molecular Probes, Invitrogen) and Hoechst 33258 (Invitrogen) for staining the actin filaments of cytoskeleton and nuclei of cells, respectively. We followed the protocols provided by the supplier, with few modifications. The specimens were observed under a fluorescence microscope (Olympus IX70, Olympus Co. Ltd., Tokyo, Japan).

Before FACS analysis each well of the 6-well plate was washed with PBS and cells released from substratum as described above. After centrifugation at $900 \mathrm{rpm}$ for 5 minutes, RBMSCs were resuspended in $0.5 \mathrm{ml}$ of complete culture medium and passed through cell strainers. ${ }^{22}$ Propidium iodide (Nacalai Tesque) was added to each sample to determine the number of live cells. Then, RBMSCs were loaded in a FACSCalibur flow cytometer (BD Biosciences Immunocytometry Systems, San Jose, California) and analyzed with a minimum of 10,000 events counting. All experiments were carried out in triplicate and following the protocol described elsewhere. ${ }^{18}$

\section{In vitro assessment of osteogenesis and viability assay}

RBMSCs (P1) were cultured in a TCPS 24-well plate at a cell density of $2 \times 10^{4}$ cells.well $^{-1}$ for 24 hours. After that time, the culture medium was replaced by the different culture medium, and RBMSCs cultured for up to 14 days. The effect of the concentration of Dex-loaded CMCht/PAMAM dendrimer NPs on the RBMSCs osteogenic differentiation was investigated as previously reported elsewhere, using positive and negative controls for osteogensesis. ${ }^{18}$ The degree of mineralization was qualitatively and quantitatively investigated as described by Uchimura et al. ${ }^{23}$ The method consists of the culturing of the RBMSCs in the presence of $1 \mu \mathrm{g} \cdot \mathrm{ml}^{-1}$ of calcein (Dojindo Laboratories, Kumamoto, Japan), which is incorporated in the mineralized ECM and allows us to investigate the calcium deposition, after 1, 7 and 14 days. The fluorescence of calcein 
incorporated into the mineralized matrices was both visualized and quantified in a image analyzer equipment (Typhoon 8600 Variable Mode Imager, Amersham Biosciences, Piscataway, New Jersey) using a $526 \mathrm{~nm}$ short pass filter. Afterward, the fluorescence of the incorporated calcein was also observed under a fluorescence microscope (Olympus IX70, Olympus Co., Ltd.). All experiments were carried out 3 times using a minimum of 4 replicates per experimental condition $(n=12)$.

\section{In vivo study}

RBMSCs were expanded in different culture media namely, MEM, MEM supplemented with Dex and MEM supplemented with the Dex-loaded CMCht/PAMAM dendrimer NPs. RBMSCs at different cell number $\left(1 \times 10^{6}\right.$ and $\left.2 \times 10^{5}\right)$ were seeded onto the surface of the SPCL scaffolds, cultured overnight in MEM complete medium for cell adhesion, and then constructs were implanted subcutaneously. Seven-week-old male F344/N rats (Syngeneic), same as donor substrain and age, were anesthetized by intraperitoneal injection of pentobarbital (Nembutal, Dainippon Pharmaceutical Co. Ltd., Osaka, Japan) at a final concentration of $3.5 \mathrm{mg}$ per $100 \mathrm{~g}$ of body weight. In each rat, 3 or 4 skin incisions (each $1 \mathrm{~cm}$ length) on the dorsal midline below the ear were made. Each RBMSCs-SPCL constructs was implanted subcutaneously ( 1.5 to $2 \mathrm{~cm}$ away from the midline at both right and left sides) into the respective pocket and skin sutured. As a negative control, we used SPCL implants without RBMSCs. All animals received their usual dietary regimen and no prophylactic medication was administered post surgery. After 4 weeks' implantation, the animals were sacrificed with an overdose of anesthetic and the implants were retrieved. All experiments were carried out 3 times using a minimum of 3 implants per condition.

\section{Micro-CT and morphometric analysis}

New bone formation in the explants was evaluated using a high-resolution micro-CT Skyscan 1072 scanner (Skyscan, Kontich, Belgium) with a resolution of pixel size of $6.59 \mu \mathrm{m}$ and integration time of $1.7 \mathrm{~ms}$. The x-ray source was set at 40 $\mathrm{keV}$ and $250 \mu \mathrm{A}$. Approximately 400 projections were acquired over a rotation range of $180^{\circ}$ with a rotation step of $0.45^{\circ}$. Data sets were reconstructed using standardized cone-beam reconstruction software (NRecon v1.4.3, SkyScan). The output format for each sample was 500 serial $1024 \times 1024$ bitmap images. A representative data set of 250 slices was segmented into binary images with a dynamic threshold of 220-255 (grey values) to assess de novo bone formation. The same representative volume of interest (VOI) was analysed for all the samples. These data sets were used for morphometric analysis (CT Analyser, v1.5.1.5, SkyScan) and to build the 3D models (ANT 3D creator, v2.4, SkyScan). The morphometric analysis included scaffold characterization such as porosity and interconnectivity, histograms and new bone formation quantification. The distribution of this new bone formation in the scaffolds was assessed by $3 \mathrm{D}$ virtual models that were created, visualized and registered using both image-processing softwares (CT Analyser and ANT 3D Creator). After $\mu-\mathrm{CT}$ analysis the explants were prepared as described in the Supplementary
Information following a standard $\operatorname{protocol}^{24}$ for further histological observation.

\section{Histological analysis}

After micro-CT analysis, the explants were decalcified with K-CX solution (Falma Co., Tokyo, Japan) for histological analysis. First, the explants were dehydrated in an ascending series grade ethanol/water solution (from $90-100 \%$ ) for 19 hours followed by washing 3 times with xylene. Then, explants were immersed in paraffin at $62^{\circ} \mathrm{C}$ and allowed to solidify at $-5{ }^{\circ} \mathrm{C}$. Slides were prepared by cutting the explants into sections $5 \mu \mathrm{m}$ thick using a microtome and mounted in a microslide glass (Matsunami Glass Ind. Ltd., Osaka, Japan). Paraffin was melt by placing the slides in the oven at $71^{\circ} \mathrm{C}$ for 20 minutes and allowed to let cool down at room temperature $\left(18-21^{\circ} \mathrm{C}\right)$. The remnant paraffin was then subsequently removed from slides by immersion in hexane for 5 minutes (S.T. Chemical Co. Ltd., Tokyo, Japan), and in a ethylene/propylene mixture (Clear Plus, Falma Co., Tokyo, Japan) for 3 minutes. Then, slides were immersed 3 times in $100 \%$ ethanol for 2 minutes each time of immersion. For the Haematoxylin \& Eosin (H\&E) staining, slides were sequentially transferred to a $90 \%$ ethanol and then to a $70 \%$ and washed with tap water. It followed the staining steps and mounted for observation as described elsewhere. ${ }^{10}$ All experiments were carried out in triplicate using 3 replicates per experimental condition $(n=9)$.

\section{Analyses of biochemical parameters}

ALP activity and osteocalcin content were measured to evaluate osteoblast differentiation in vivo. Before the assay the explants were washed with $\mathrm{Ca}$ and $\mathrm{Mg}$-free PBS solution. Then, explants were processed as previously reported. ${ }^{19,24}$ Standards were prepared with p-nytrophenol, $\mathrm{pNP}$. Triplicates were made for each sample and standard and experiments repeated 3 times $(n=9)$. Absorbance was read at $405 \mathrm{~nm}$ (Wallac ARVOsx 1420, Perkin-Elmer Life and Analytical Sciences), and sample concentrations were read from the standard graph. Enzyme activity was expressed either as nmol of pNP released/explant/30 minutes. The remnant of each sample used for the ALP assay of the explants was treated with a $20 \%$ formic acid solution and stored at $4^{\circ} \mathrm{C}$ for $2-3$ days. Afterward samples were centrifuged at $15,000 \mathrm{rpm}$ and the supernatant was passed through a Sephadex G-25 column (GE Healthcare, Uppsala, Sweden). The samples were subsequently concentrated for performing the enzyme-linked immunosorbent assay (ELISA). A rat osteocalcin EIA kit ( $\mathrm{N}^{\circ}$ BT-460, Biomedical Technologies Inc., Stoughton, Massachusetts) was used following the instructions provided by the supplier. Data were read off from the standard curve obtained with human osteocalcin and expressed as ng of deposited osteocalcin per explant. As an index of mineral bone formation, deposited calcium in the explants was quantified as described by Kim et $\mathrm{al}^{25}$ with minor modifications. Statistical analysis was carried out as described in the Supplementary Information.

For calcium determination, explants were rinsed with $\mathrm{Ca}$ and $\mathrm{Mg}$-free PBS solution and $0.2 \mathrm{ml}$ of $1 \mathrm{~N} \mathrm{HCl}$ solution per explant was added. The tissues were chopped before being placed on an orbital shaker to extract calcium for 12 hours. Then, mixtures 

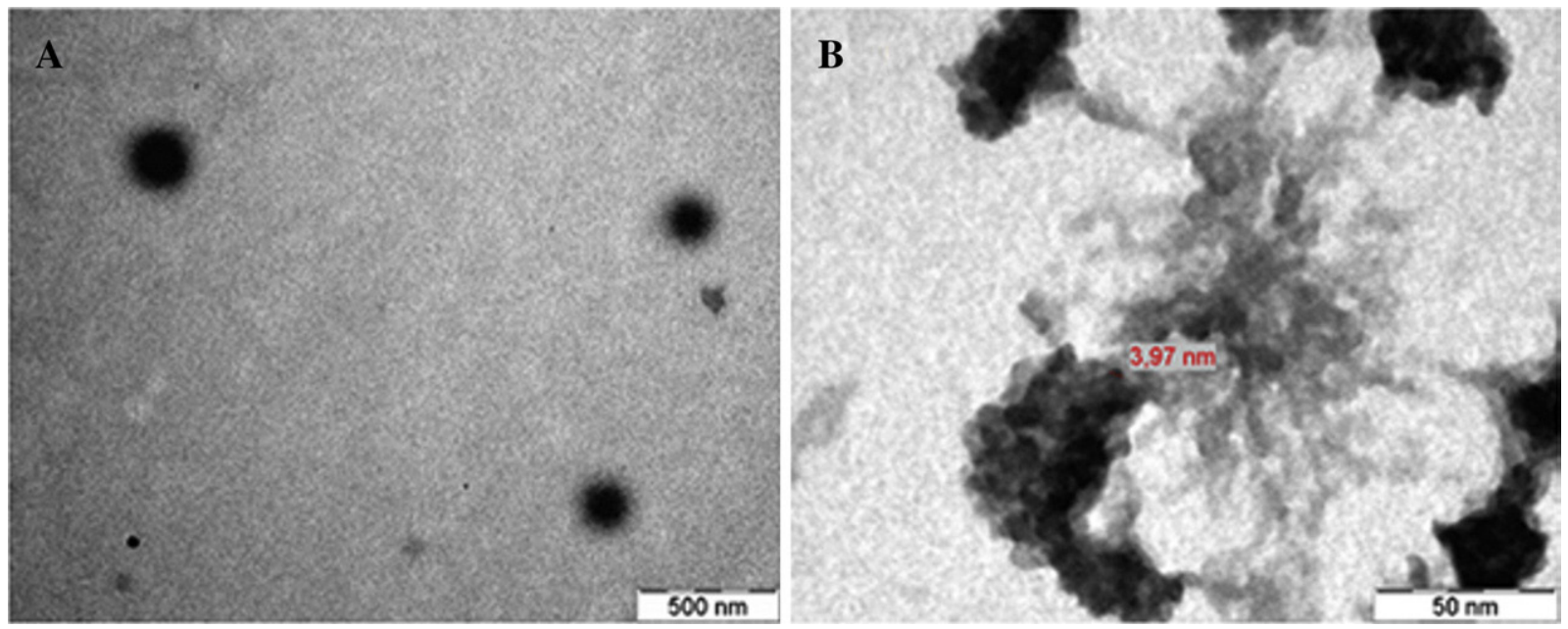

Figure 1. Characterization of the nanoparticles. Representative TEM images of the CMCht/PAMAM dendrimer NPs (A), and Dex-loaded CMCht/PAMAM dendrimer NPs (B).

were centrifuged at 15,000 rpm for 10 minutes and supernatants were assayed (o-cresolphthalein complexation color development method) using a commercial calcium assay kit (Calcium Ctest, Wako Pure Chemical Industries, Osaka, Japan). Calcium content was determined by measuring the absorbance at $570 \mathrm{~nm}$. All experiments were carried out in triplicate using 3 replicates per experimental condition $(\mathrm{n}=9)$.

\section{Statistical analysis}

Statistical differences were analysed using the GraphPad Prism software (GraphPad Software Inc., La Jolla, California). One-way analysis of variance (Tukey's multiple comparison test) was performed and statistical significance was defined as $P<0.05$ for a $95 \%$ confidence interval $(P$ value: $* * *<$ $0.001 ; * *<0.01 ; *<0.05)$.

\section{Results}

In the present work, ${ }^{1} \mathrm{H}$ NMR analysis was carried to investigate the loading of Dex into the Dex-loaded CMCht/ PAMAM dendrimer NPs (Figure S1). The spectrum shows the typical signals from CMCht, PAMAM dendrimers and Dex. It is possible to observe small singlets at $1.14,1.28 \mathrm{ppm}$ and 1.86 ppm from $\mathrm{CH}_{3}$ and $\mathrm{CH}_{2}$ groups, and a doublet at $7.55 \mathrm{ppm}$ and $7.96 \mathrm{ppm}$, which are attributed to the chemical shifts of the protons attached to $\mathrm{C} 1$ atoms and to aromatic ring (*) of Dex, respectively. By its turn, to evaluate the morphology of the NPs upon loading with Dex, TEM analysis was performed (Figure 1). From images we can observe that the loading of Dex can affect the morphology of the NPs. In fact the formation of dendronlike NPs instead of the typical nanosphere-like morphology is revealed. Zeta potential and particle size of the CMCht/ PAMAM dendrimer NPs and Dex-loaded CMCht/PAMAM dendrimer NPs were also measured by DLS (Table S1). Results showed that the hydrodynamic diameter of the CMCht/PAMAM dendrimer NPs in PBS ( $\mathrm{pH} 7.4$ ), increased upon Dex loading, and the value of the NPs' zeta potential changed from -32.8 to $-27.3 \mathrm{mV}$ (experiments carried out in water).

In vitro cell culture studies were performed to investigate possible cytotoxicity and uptake of NPs by RBMSCs. Fluorescence microscopy images of RBMSCs cultured in the presence of FITC-labeled and Dex-loaded CMCht/PAMAM dendrimer NPs revealed that the NPs are taken up by RBMSCs, after 12 hours (Figure 2). The cell-viability assay of RBMSCs cultured in the presence of different culture medium revealed that NPs are noncytotoxic over RBMSCs at concentrations bellow $1 \mathrm{mg} . \mathrm{ml}^{-1}$ (Figure S2). FACS analysis was also performed after culturing the RBMSCs in the presence of the FITC-labelled NPs for up to 14 days (Figure S3 and Table S2). FACS analysis revealed increasing levels of fluorescence associated with cells after incubation of RBMSCs with CMCht/PAMAM dendrimer NPs. The fraction of live cells cultured in the presence of FITC-labeled Dex-loaded CMCht/PAMAM dendrimer NPs do not significantly differ from that of controls. This is a good indication that NPs do not elicit any cytotoxic effect over RBMSCs. The osteogenic ability of Dex-loaded CMCht/PAMAM dendrimer NPs was investigated by qualitatively and quantitatively determining the levels of calcein uptake (Figure 3 and Figure S4). Results demonstrated that mineralization can only occur in cultures in which RBMSCs were supplemented with Dex-loaded CMCht/PAMAM dendrimer NPs in comparison with to positive control (osteogenic medium). Moreover, it can be seen that NP concentration significantly affects mineralization, i.e., superior mineralization occurs when using $0.01 \mathrm{mg} \cdot \mathrm{ml}^{-1}$ Dex-loaded CMCht/PAMAM dendrimer NPs in comparison with that for $1 \mathrm{mg} . \mathrm{ml}^{-1}$ Dex-loaded CMCht/PAMAM dendrimer NPs. The osteogenic studies are supported by phase contrast and respective fluorescence microscopy images of RBMSCs after culturing in different culture medium and in the presence of calcein for 14 days (Figure S5). In the Figures 5S, $C-E$, it can be observed the typical mineral nodule formation in RBMSCs cultures that were exposed to Dex, both from culture media or from the Dex-loaded CMCht/PAMAM dendrimer NPs. 

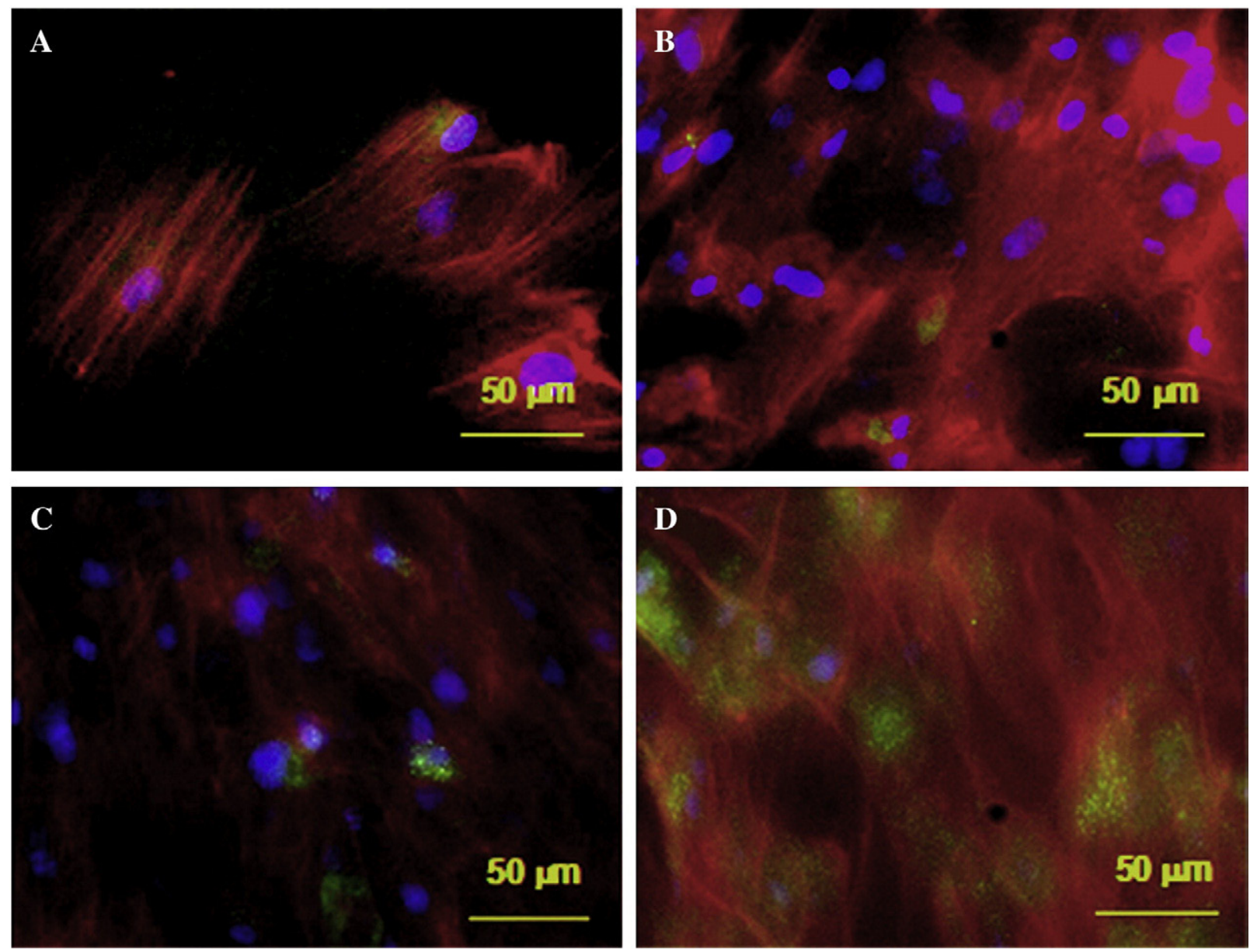

Figure 2. Cellular uptake of the dendronlike NPs. Fluorescence microscopy images of the RBMSCs cultured in the presence of FITC-labelled Dex-loaded CMCht/PAMAM dendrimer NPs 0.1 mg.ml ${ }^{-1}$ (green) for: $12 \mathrm{~h}$ (A) $24 \mathrm{~h}$ (B) $7 \mathrm{~d}$ (C) and $14 \mathrm{~d}$ (D). Nuclear DNA and cytoskeleton were labeled with Hoechst 33258 (blue) and Texas-red phalloidin (red), respectively.

In in vivo experiments, the ability of the Dex-loaded CMCht/ PAMAM dendrimer NPs in the production of new bone was evaluated using RBMSCs seeded onto the SPCL scaffolds. The effect of cell number on the bone-forming ability was investigated. No signs of infection or acute inflammatory reaction were detected at the implantation sites for all implants after 4 weeks of implantation (Figure S6). Figure 4 shows the 3D micro-CT images and respective $\mathrm{H} \& \mathrm{E}$ stained sections of the explants. Further 3D micro-CT morphometric analysis can be seen in Supplementary Information (Figures S7-S9). Moreover, ALP activity, osteocalcin and calcium contents of the explants were also quantitatively investigated. Results demonstrated that cell density and culture conditions greatly affect in vivo bone formation. From data, it can be seen that bone formation occurs when seeding the SPCL scaffolds with $1 \times 10^{6}$ RBMSCs exposed to Dex either from culture medium and Dex-loaded CMCht/PAMAM dendrimer NPs during expansion ex vivo. The biochemical data corroborated the histological and micro-CT findings. A significant increase $(P<0.001)$ in the calcium deposited is observed culturing RBMSCs with Dex-loaded CMCht/PAMAM dendrimer NPs in comparison with explants seeded with $1 \times 10^{6}$ RBMSCs exposed to Dex from culture medium.

\section{Discussion}

The water-soluble NPs were characterized by NMR, DLS and TEM. The ${ }^{1} \mathrm{H}$ NMR spectra of CMCht/PAMAM dendrimer NPs and Dex-loaded CMCht/PAMAM dendrimer NPs are provided as Supplementary Information (Figure S1). The spectrum of the Dex-loaded CMCht/PAMAM dendrimer NPs shows the typical signals from CMCht, PAMAM dendrimers and Dex. It is possible to observe the chemical shifts of the protons attached to $\mathrm{C} 1$ atoms and to aromatic ring of Dex, which clearly shows that Dex $^{26}$ was effectively loaded into the CMCht/PAMAM dendrimer NPs. ${ }^{24}$ This data corroborate previous findings, ${ }^{18}$ which showed that Dex can be loaded into the NPs and released as free drug. The release kinetics of Dex follow a steady release after the initial burst ( 24 hours), which can last for 7 days. In fact, experiments performed in PBS with $15 \% \mathrm{FBS},{ }^{18}$ revealed that Dex concentration reaches a plateau of $4 \mu \mathrm{g} \cdot \mathrm{ml}^{-1}$. This data revealed that by adding the Dex-loaded CMCht/PAMAM dendrimer NPs to culture media at a final concentration of $0.01 \mathrm{mg} \cdot \mathrm{ml}^{-1}$, the concentration of Dex is expected to be in the magnitude of $10 \mathrm{~nm}$. The time scale for the Dex release from the NPs was observed to be in the same order of their internalization 


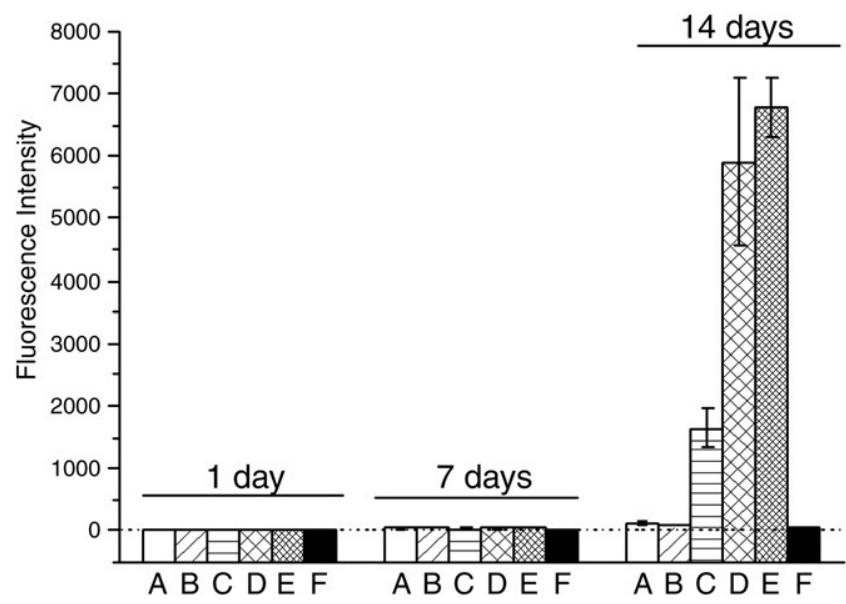

Figure 3. In vitro osteogenic ability of the dendron-like NPs. Fluorescence intensity values of RBMSCs cultured in the presence of calcein, for the period of 1-14 days. Values were related to fluorescence intensity of RBMSCs cultured in the absence of calcein, for each condition. (A) MEM medium (negative control); (B) $1 \mathrm{mg} \cdot \mathrm{ml}^{-1} \mathrm{CMCht} / \mathrm{PAMAM}$ dendrimer NPs; (C) 1 mg.ml ${ }^{-1}$ Dex-loaded CMCht/PAMAM dendrimer NPs; (D) $0.01 \mathrm{mg} \cdot \mathrm{ml}^{-1}$ Dex-loaded CMCht/PAMAM dendrimer NPs; (E) Osteogenic medium, and (F) MEM medium with $\beta$-glycerophosphate. Results are expressed as mean \pm s.d. $(n=12)$. The Tukey's multiple comparison test was used for statistical analysis.

by cells. Figure $1, A$ shows the TEM images of the CMCht/ PAMAM dendrimer NPs that exhibit a spherical shape. We can also observe that the loading of Dex can affect the morphology of the NPs (Figure 1, B). In fact, it is possible to observe the formation dendron-like NPs, instead of the typical nanospherelike morphology. To investigate the effect of $\mathrm{pH}$ on the particle size and distribution, and zeta potential DLS analysis were performed. These studies were carried out at physiological and lysosomal typical pH's (see Table S1). The results show that upon loading of Dex into the CMCht/PAMAM dendrimer NPs, the hydrodynamic diameter of the particles and polydispersivity increased, and no significant changes on the zeta potential was observed at $\mathrm{pH}$ 7.4. However, when the DLS studies were carried out in water, the value of zeta potential of the NPs changed from -32.8 to $-27.3 \mathrm{mV}$, upon loading of Dex. In an acidic medium, the amino and carboxylic groups of $\mathrm{CMCht}$ are protonated. Thus, the zeta potential of the NPs at $\mathrm{pH} 3$ loosed the strong negative character, as expected. In fact at slightly acidic, the NPs will possess a zero charge. This would mean that when internalized by cells and inside the lysosomes $(\mathrm{pH}<5.5)$, as the NPs possess a significant buffering capacity, it can act as "proton sponges". ${ }^{27}$ Therefore, we can hypothesize that the neutralization of the NPs' surface charge at acidic $\mathrm{pH}$ 's can cause coagulation of the particles and prevent Dex release within lysosomes. Actually, the charge conversion may be beneficial for lysosomal escape and subsequently release Dex in the cytoplasm, where it can bind and activate the glucocorticoid receptor. This behavior may open other application possibilities namely, the use of these nanocarriers in gene delivery. However, these statements remain to be clarified and studies in these directions will be reported elsewhere.
As mentioned, particle size can affect internalization by cells. On the other hand, in a report by Zhang et $\mathrm{al}^{28}$ it was demonstrated that Dex treatment decreases the phagocytosis of Staphylococcus aureus bioparticles, in normal primary trabecular meshwork cells. To further investigate if the incorporation of Dex into the NPs may affect the internalization ability of RBMSCs, a FITC probe was linked to these NPs. Fluorescence microscopy images of RBMSCs cultured in the presence of FITC-labeled and Dex-loaded CMCht/PAMAM dendrimer NPs revealed that the NPs are taken up by RBMSCs, after 12 hours (Figure 2). RBMSCs continued to internalize the NPs (higher fluorescent signals), when maintained for longer culturing periods. These data are corroborated by the cell viability study (see Figure S2), demonstrating the noncytotoxicity of Dexloaded CMCht/PAMAM dendrimer NPs. Fluorescence-activated cell sorter (FACS) analysis was also performed after culturing the RBMSCs in the presence of the FITC-labeled NPs for up to 14 days (Figure S3 and Table S2). FACS analysis revealed increasing levels of fluorescence associated with cells after incubation RBMSCs with CMCht/PAMAM dendrimer NPs. The fraction of live cells cultured in the presence of FITC-labelled Dex-loaded CMCht/PAMAM dendrimer NPs do not significantly differ from that of controls. This is a good indication that NPs do not elicit any cytotoxic effect over RBMSCs.

The later stages of the osteogenic differentiation of mesenchymal stem cells give rise to cells able to produce mineralized extracellular matrix (ECM). The osteogenic ability of Dex-loaded CMCht/PAMAM dendrimer NPs was investigated by qualitatively and quantitatively determining the levels of calcein uptake. This method allows monitoring the calcein fluorescence arising from the mineralization process, ${ }^{23}$ which denotes the osteogenic activity in a culture (Figure S4). It is possible to observe that mineralization can only occur in cultures in which RBMSCs were supplemented with Dex-loaded CMCht/ PAMAM dendrimer NPs and osteogenic medium. Figure 3 shows the fluorescence intensity values of RBMSCs cultured in the presence of calcein. These results revealed that no significant differences in mineralization were observed for cultures supplemented with $0.01 \mathrm{mg} \cdot \mathrm{ml}^{-1}$ Dex-loaded CMCht/PAMAM dendrimer NPs in comparison with that for osteogenic medium. This is a good indication that the NPs possess osteogenic ability within this range of concentrations. By its turn, the calcein uptake, which denotes the degree of mineralization, is decreased in cultures supplemented with Dex-loaded CMCht/PAMAM dendrimer NPs at a concentration of $1 \mathrm{mg} \cdot \mathrm{ml}^{-1}$. This data indicates that the NPs' concentration is not the optimal level to allow the supply of Dex within the optimal range of concentrations to maximize the osteogenesis. These data are not surprising, because it has been reported ${ }^{29,30}$ that optimal concentration $\left(10^{-8} \mathrm{M}\right)$ and regimented supply of Dex is needed for promoting the differentiation of RBMSCs towards osteoblast over adipocytes. These results are supported by phase contrast (left) and respective fluorescence microscopy images of RBMSCs after culturing in different culture media and in the presence of calcein for 14 days (see Figure S5).

In in vivo experiments (Figure 4), the ability of the Dexloaded CMCht/PAMAM dendrimer NPs toward the production of new bone was evaluated using SPCL scaffolds with $67.4 \pm$ 
SPCL

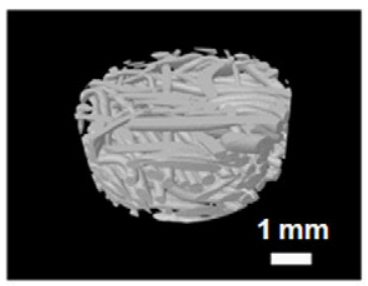

SPCL explant

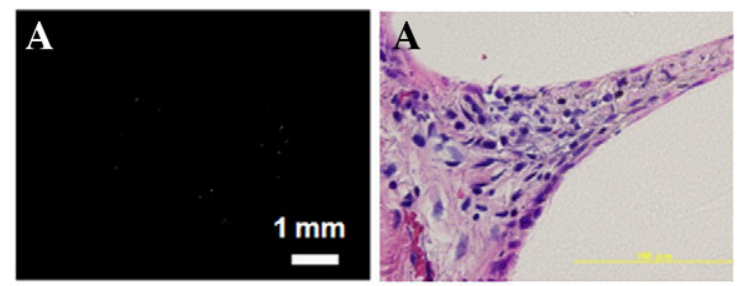

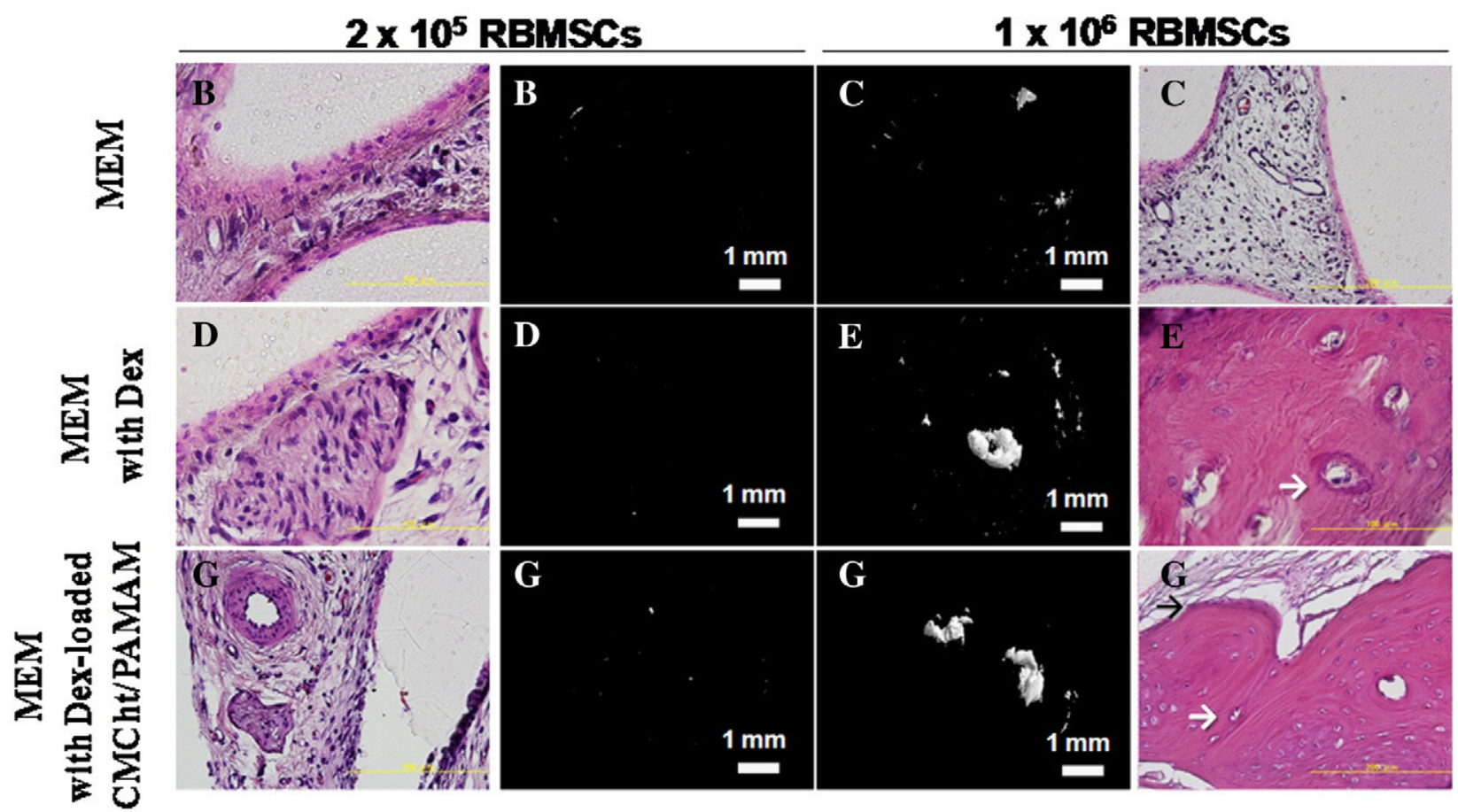
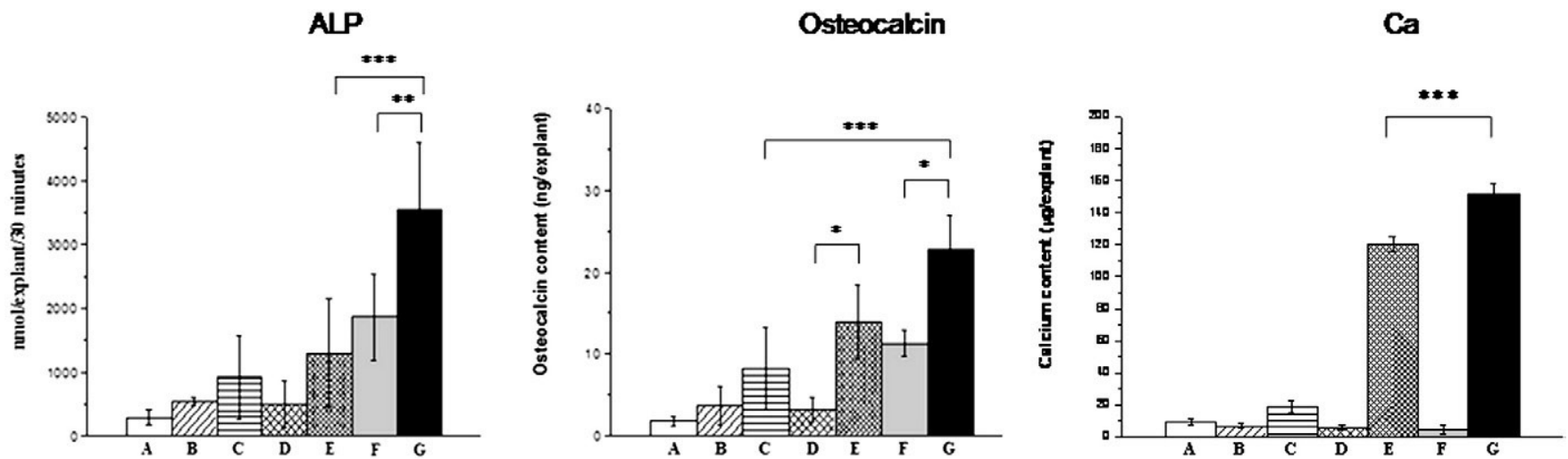

Figure 4. In vivo de novo bone formation ability of the dendronlike NPs. 3D micro-CT images and respective H \& E stained sections of the explants. ALP activity, osteocalcin and calcium contents of the explants after 4 weeks of subcutaneous implantation: SPCL scaffold explants (A) explants with $2 \times 10^{5}$ RBMSCs cultured in MEM (B) explants with $1 \times 10^{6}$ RBMSCs cultured in MEM (C) explants with $2 \times 10^{5}$ RBMSCs cultured in MEM with Dex (D) explants with $1 \times 10^{6}$ RBMSCs cultured in MEM with Dex (E) explants with $2 \times 10^{5}$ RBMSCs cultured in MEM with Dex-loaded CMCht/PAMAM dendrimer NPs (F) and explants with $1 \times 10^{6}$ RBMSCs cultured in MEM with Dex-loaded CMCht/PAMAM dendrimer NPs (G). Results are expressed as mean \pm Standard deviation $(\mathrm{SD})(\mathrm{n}=9)$. The Tukey's multiple comparison test was used for statistical analysis $(P$ value: $* * *<0.001 ; * *<0.01 ; *<0.05)$.

$1.3 \%$ porosity (as determined by micro-CT) as the $3 \mathrm{D}$ matrix. These were chosen because we found previously ${ }^{19}$ that SPCL is an adequate support for the RBMSCs osteogenic differentiation in vitro. The SPCL scaffolds also exhibits lower attenuation coefficient than that for bone or calcium phosphates, i.e., the intensity of the $\mathrm{x}$-ray beam is reduced less as it passes through the 


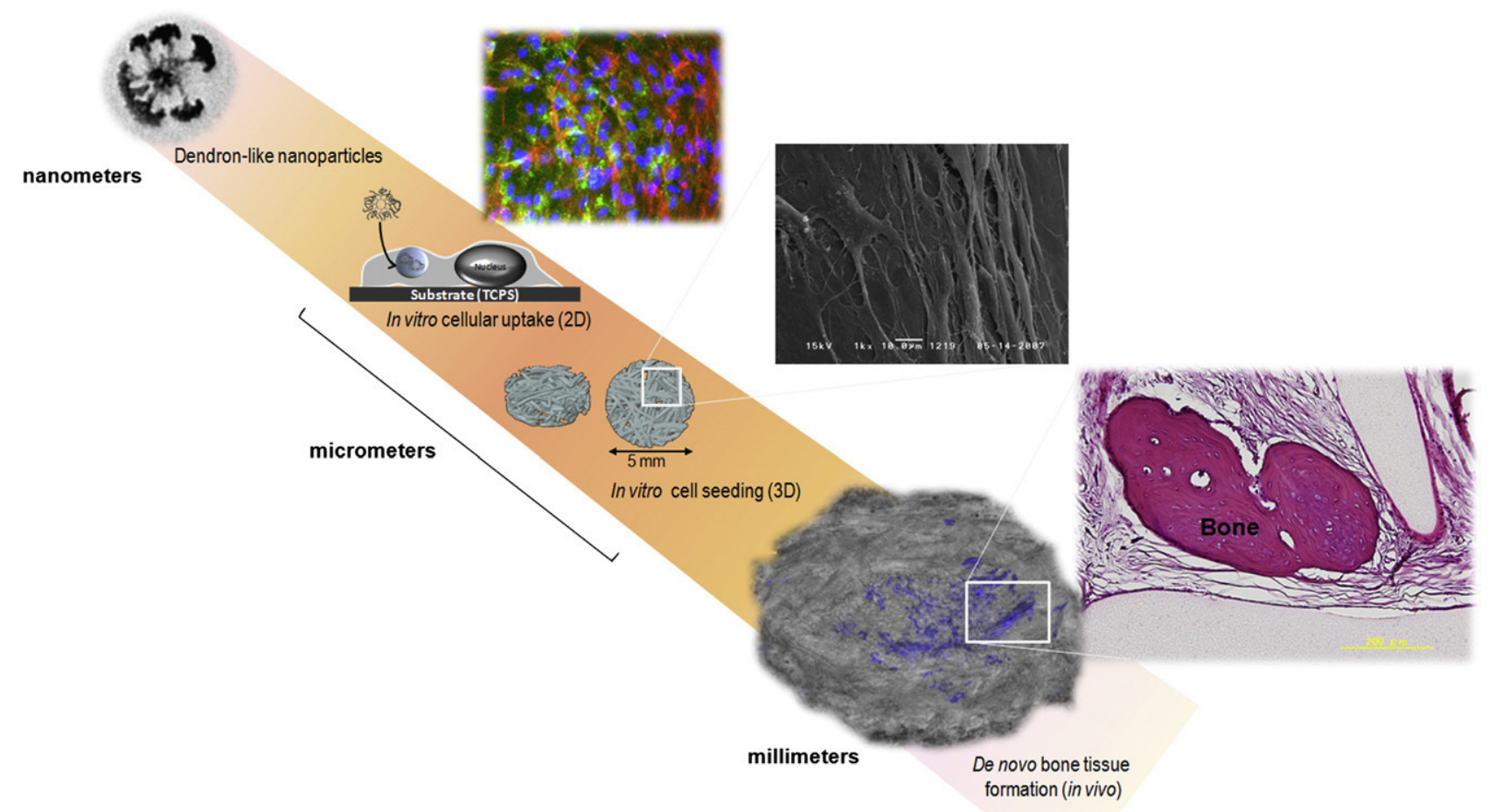

Figure 5. Schematic illustration of ex vivo stem cells "tune-up" and de novo bone tissue formation in vivo. Dendronlike NPs are internalized by stem cells in vitro, and then cells are seeded onto SPCL scaffolds and implanted. Release of Dex to the cytoplasm will occur and de novo bone formation (blue areas) is observed within SPCL scaffolds after 4 weeks of implantation.

SPCL material. ${ }^{21}$ Therefore, it would be possible to determine the total volume of newly formed bone into implants by using this technique. ${ }^{31}$ We cultured in vitro the RBMSCs in the presence of $0.01 \mathrm{mg} \cdot \mathrm{ml}^{-1}$ Dex-loaded CMCht/PAMAM dendrimer NPs until reaching confluence, seeded the RBMSCs onto the surface of the SPCL scaffolds overnight followed by subcutaneous implantation into the back of F344/N rats for 4 weeks. The effect of cell number on the bone-forming ability was also investigated because we have reported that it may influence de novo bone formation. ${ }^{24}$ No signs of infection or acute inflammatory reaction were detected at the implantation sites for all implants after 4 weeks of implantation (see Figure S6). From micro-CT analyses of the explants namely, SPCL alone and SPCL scaffolds seeded with $2 \times 10^{5}$ and $1 \times 10^{6}$ RBMSCs (explants) whose cells were expanded in MEM medium, no areas of high attenuation were observed (Figure $4, A-C$ ). On the contrary, it was possible to observe regions of higher attenuation between 0.7 and $0.8 \mathrm{~cm}^{-1}$ (black arrow) in the constructs seeded with $1 \times 10^{6}$ RBMSCs that were exposed to Dex and Dex-loaded CMCht/PAMAM dendrimer NPs (Figure 4, $E$ and $G$; also see Figure S7). The areas of higher attenuation detected can be an indication of de novo bone formation because the SPCL scaffolds and SPCL explants have an attenuation coefficient between 0.3 and $0.6 \mathrm{~cm}^{-1}$, which is lower than that for ceramics and mineralized matrices. To confirm such hypothesis, histological and biochemical studies were carried out. Figure 4 (top) shows the 3D images of the SPCL scaffolds and explants obtained from micro-CT analyses and respective microscopic images of the explants histological sections stained with $\mathrm{H} \& \mathrm{E}$. By analyzing the images after imposing a threshold to evidence the high-density regions, it is possible to observe that the region of higher attenuation observed corresponds to the white area in the 3D micro-CT images (see Figures S7-S8). In fact, compact masses (white area) were only observed in the explants whose RBMSCs were exposed to Dex and Dex-loaded CMCht/ PAMAM dendrimer NPs during expansion, in vitro (Figure 4, $E$ and $G)$. No bone formation in the explants of SPCL alone (control) and SPCL scaffolds seeded with $2 \times 10^{5}$ RBMSCs was observed (Figure 4, $A-B$ ). From the histological photographs of the explants, it became evident that the massive area of high attenuation (white area) corresponds to newly formed bone. By its turn, explants whose RBMSCs were not exposed to Dex, fibrous tissue formation within the pores of SPCL scaffolds was observed. The superior bone organization in the explants whose RBMSCs were supplemented with Dex-loaded CMCht/ PAMAM dendrimer NPs in comparison with that whose RBMSCs were supplemented with Dex from culture media might be one of the most important achievements in this study. Cuboid osteoblasts lining the forming bone matrix in SPCL scaffolds seeded with $1 \times 10^{6}$ RBMSCs indicate an active bone formation site (black arrow) (Figure 4, $G$ ). It is also possible to observe the lacunar spaces and reminiscent osteocyte-like cells entrapped in the bone matrix (white arrows). Thus, osteocytic lacunae in the lamellar bone are uniform and regularly 
distributed with osteoblasts forming a continuous layer of bone in a unidirectional way typical of mature bone. Moreover, we observed that new bone formation occurs primarily at the middle section of the scaffold (see Figure S8B), which reveals that the scaffolds possess an adequate pore size and interconnectivity. Micro-CT analyses also revealed that in the explants with $1 \times 10^{6}$ RBMSCs cultured in MEM media, bone formation occurs though in low amounts. To evaluate further the osteogenic differentiation in vivo, the alkaline phosphatase (ALP) activity was measured (Figure 4, bottom left). Results demonstrated that ALP activity significantly increased in the explants seeded with $1 \times 10^{6}$ RBMSCs that were exposed to Dex-loaded CMCht/ PAMAM dendrimer NPs as compared to either, explants seeded with $2 \times 10^{5}$ RBMSCs exposed to Dex-loaded CMCht/PAMAM dendrimer NPs and explants seeded with $1 \times 10^{6}$ RBMSCs exposed to Dex supplemented from culture media. These data are not surprising because the previous in vitro release study ${ }^{18}$ of Dex from the CMCht/PAMAM dendrimer NPs showed that Dex concentration resembles that from osteogenic media. This article, however, reports for the first time that SPCL scaffolds are effective in supporting the osteogenic differentiation of RBMSCs and de novo bone formation in vivo. Figure 4 (bottom center) shows the content of the late osteogenic marker and bonespecific osteocalcin in the explants after 4 weeks of implantation. No significant differences in osteocalcin levels were seen for SPCL explants without cells and seeded with $2 \times 10^{5}$ RBMSCs cultured in MEM and MEM supplemented with Dex. Although there is an statistical increase $(P<0.05)$ in osteocalcin levels for the explants seeded with $1 \times 10^{6}$ RBMSCs exposed to Dexloaded CMCht/PAMAM dendrimer NPs in comparison with that of the explants seeded with $2 \times 10^{5}$ RBMSCs cultured in the same culture conditions. Moreover, a significant increase of the osteocalcin content can be observed in the explants seeded with $1 \times 10^{6}$ RBMSCs exposed to Dex in comparison with that of the explants seeded with $2 \times 10^{5}$ RBMSCs. This data revealed that cell number significantly affects this late osteogenic marker in vivo. Significant differences in the organization of the newly formed bone were observed under the histological studies. To elucidate these findings, calcium was extracted from the explants by acid treatment to investigate the quality and degree of mineralization of the newly bone formed (Figure 4, bottom right). No considerable calcium deposition was found in the explants of SPCL scaffold and SPCL scaffolds seeded with the RBMSCs that were cultured in the absence of Dex. These results corroborates the histological and micro-CT findings which demonstrated that new bone formation only occur in the explants seeded with $1 \times 10^{6}$ RBMSCs exposed to Dex and Dex-loaded CMCht/ PAMAM dendrimer NPs. A significant increase $(P<0.001)$ in the calcium deposited is observed when expanding the RBMSCs with Dex-loaded CMCht/PAMAM dendrimer NPs ain comparison with to explants seeded with $1 \times 10^{6}$ RBMSCs exposed to Dex, which in its turn denoted the superior mineralization of the newly formed bone. We advance a possible explanation for the superior bone organization and mineralization observed in the explants whose RBMSCs were cultured with Dex-loaded CMCht/PAMAM dendrimer NPs. This can be due to the supply of Dex for longer time and in a regimented manner, as Dex possibly can be released from the NPs inside cells during in vivo implantation time. However, further studies need to be carried out to clarify this issue and to investigate the interaction of Dex with the GC receptors and its translocation into the nucleus. The biochemical data shows that the acellular approach, i.e., SPCL scaffolds alone, does not induce in vivo de novo bone formation. However, by seeding RBMSCs supplemented with Dex onto the SPCL scaffolds, bone formation can be observed when cell density was $1 \times 10^{6}$ cells per scaffold. The SPCL scaffolds proved to be adequate for fundamental studies as it supported new bone formation while allowing us to quantitatively assess new bone formation in vivo, thus satisfying the requirements of a temporary scaffold for the effective stem cells "tune-up" (Figure 5), and to be used in basic studies.

In summary, the synthesized dendron like Dex-loaded CMCht/PAMAM dendrimer NPs were found to possess a $\mathrm{pH}$ and ionic-responsive nature. The NPs were able to be internalized by cells and to promote the osteogenic differentiation of RBMSCs in vitro. The present work demonstrated the use of noncytotoxic NPs able to modulate the differentiation pathway from the interior of stem cells, being able, in particular to promote superior bone formation in vivo, when combining such stem cell-based strategies and tissue engineering approaches. This work clearly evidences the advantage of using intracellular nanocarrier tools for preprogramming the stem cells ex vivo as these NPs show promise in the development of new bone tissue.

\section{Appendix A. Supplementary data}

Supplementary data to this article can be found online at doi:10.1016/j.nano.2011.03.002.

\section{References}

1. Lutolf MP, Hubbell JA. Synthetic biomaterials as instructive extracellular microenvironments for morphogenesis in tissue engineering. Nat Biotechnol 2005;23:47-55.

2. Bianco P, Robey PG. Stem cells in tissue engineering. Nature 2001;414: 118-21.

3. Dalby MJ, Gadegaard N, Tare R, Andar A, Riehle MO, Herzyk P, et al. The control of human mesenchymal cell differentiation using nanoscale symmetry and disorder. Nat Mater 2007;6:997-1003.

4. Kraljevic S, Pavelic K. Navigare necessere est. EMBO reports 2005;6:695-700.

5. Wang Y, Gao S, Ye W-H, Yoon HS, Yang Y-Y. Co-delivery of drugs and DNA from cationic core-shell nanoparticles self-assembled from a biodegradable copolymer. Nat Mater 2006;5:791-6.

6. Nishiyama N, Iriyama A, Jang W-D, Miyata K, Itaka K, Inoue Y, et al. Light-induced gene transfer from packaged DNA enveloped in a dendrimeric photosensitizer. Nat Mater 2005;4:934-41.

7. You M-H, Kwak MK, Kim D-H, Kim K, Levchenko A, Kim D-Y, et al. Synergistically enhanced osteogenic differentiation of human mesenchymal stem cells by culture on nanostructured surfaces with induction media. Biomacromolecules 2010;11:1856-62.

8. Neu M, Germershaus O, Mao S, Voigt K-H, Behe M, Kissel T. Crosslinked nanocarriers based upon poly(ethylene imine) for systemic plasmid delivery: in vitro characterization and in vivo studies in mice. J Control Release 2007;118:370-80. 
9. Kocbek P, Obermajer N, Cegnar M, Kos J, Kristl J. Targeting cancer cells using PLGA nanoparticles surface modified with monoclonal antibody. J Control Release 2007;120:18-26.

10. Lee CC, Gillies ER, Fox ME, Guillaudeu SJ, Fréchet JMJ, Dy EE, et al. A single dose of doxorubicin-functionalized bow-tie dendrimer cures mice bearing C-26 colon carcinomas. Proc Natl Acad Sci U S A 2006;103:16649-54.

11. Kano MR, Bae Y, Iwata C, Morishita Y, Yashiro M, Oka M, et al. Improvement of cancer-targeting therapy, using nanocarriers for intractable solid tumors by inhibition of TGF- ${ }^{2}$ signaling. Proc Natl Acad Sci U S A 2007;104:3460-5.

12. Medintz IL, Uyeda TH, Goldman ER, Mattoussi H. Quantum dot bioconjugates for imaging, labelling and sensing. Nat Mater 2005;4:435-46.

13. Tsogas I, Sideratou Z, Tsiourvas D, Theodossiou TA, Paleos CM. Interactive transport of guanidinylated poly(propylene-imine)-based dendrimers through liposomal and cellular membranes. Chem Bio Chem 2007;8:1865-76.

14. Kukowska-Latallo JF, Bielinska AU, Johnson J, Spindler R, Tomalia DA, Baker JR. Efficient transfer of genetic material into mammalian cells using Starburst polyamidoamine dendrimers. Proc Natl Acad Sci U S A 1996;93:4897-902.

15. Malik N, Wiwattanapatapee R, Klopsch R, Lorenz K, Frey H, Weener JW, et al. Dendrimers: relationship between structure and biocompatibility in vitro, and preliminary studies on the biodistribution of ${ }^{125}$ I-labelled polyamidoamine dendrimers in vivo. J Control Release 2000;65:133-48.

16. Lee CC, MacKay JA, Fréchet JM, Szoka FC. Designing dendrimers for biological applications. Nat Biotechnol 2005;23:1517-26.

17. Kobayashi H, Kawamoto S, Jo S-K, Sato N, Saga T, Hiraga A, et al. Renal tubular damage detected by dynamic micro-MRI with a dendrimerbased magnetic resonance contrast agent. Kidney Int 2002;61:1980-5.

18. Oliveira JM, Kotobuki N, Marques AP, Pirraco RP, Benesch J, Hirose M, et al. Surface engineered carboxymethylchitosan/poly (amidoamine) dendrimer nanoparticles for intracellular targeting. Adv Funct Mater 2008;18:1840-53.

19. Oliveira JM, Sousa RA, Kotobuki N, Tadokoro M, Hirose M, Mano JF, et al. The osteogenic differentiation of rat bone marrow stromal cells cultured with dexamethasone-loaded carboxymethylchitosan/polyamidoamine dendrimer nanoparticles. Biomaterials 2009;30:804-13.

20. Sashiwa H, Shigemasa Y, Roy R. Chemical modification of chitosan 8: preparation of chitosan-dendrimer hybrids via short spacer. Carbohydr Polym 2002;47:191-9.
21. Oliveira AL, Malafaya PB, Costa SA, Sousa RA, Reis RL. Microcomputed tomography ( $\mu$-CT) as a potential tool to assess the effect of dynamic coating routes on the formation of biomimetic apatite layers on 3D-plotted biodegradable polymeric scaffolds. J Mater Sci: Mater Med 2007;18:211-23.

22. Kotobuki N, Hirose M, Takakura Y, Ohgushi H. Cultured autologous human cells for hard tissue regeneration: preparation and characterization of mesenchymal stem cells from bone marrow. Artif Organs 2004;28:33-9.

23. Uchimura E, Machida H, Kotobuki N, Kitamura T, Ikeuchi M, Hirose M, et al. In-situ visualization and quantification of mineralization of cultured osteogenetic cells. Calcif Tissue Int 2003;73:575-83.

24. Oliveira JM, Kotobuki N, Tadokoro M, Hirose M, Mano JF, Reis RL, et al. Ex vivo culturing of stromal cells with dexamethasone-loaded carboxymethylchitosan/poly(amidoamine) dendrimer nanoparticles promotes ectopic bone formation. Bone 2010;46:1424-35.

25. Kim H, Kim HW, Suh H. Sustained release of ascorbate-2-phosphate and dexamethasone from porous PLGA scaffolds for bone tissue engineering using mesenchymal stem cells. Biomaterials 2003;24: 4671-9.

26. Midelfart A, Dybdahl A, Muller N, Sitter B, Gribbestad IS, Krane J. Dexamethasone and dexamethasone phosphate detected by ${ }^{1} \mathrm{H}$ and ${ }^{19} \mathrm{~F}$ NMR spectroscopy in the aqueous humour. Exp Eye Res 1998;66:327-37.

27. Boussif O, Lezoualc'h F, Zanta MA, Mergny MD, Scherman D, Demeneix B, et al. A versatile vector for gene and oligonucleotide transfer into cells in culture and in vivo: Polyethylenimine. Proc Natl Acad Sci U S A 1995;92:7297-301.

28. Zhang X, Ognibene CM, Clark AF, Yorio T. Dexamethasone inhibition of trabecular meshwork cell phagocytosis and its modulation by glucocorticoid receptor b. Exp Eye Res 2007;84:275-84.

29. Beresford JN, Bennett JH, Devlin C, Leboy PS, Owen ME. Evidence for an inverse relationship between the differentiation of adipocytic and osteogenic cells in rat bone marrow stromal cell cultures. J Cell Sci 1992;102:341-51.

30. Porter RM, Huckle WR, Goldstein AS. Effect of dexamethasone withdrawal on osteoblastic differentiation of bone marrow stromal cells. J Cell Biochem 2003;90:13-22.

31. Mastrogiacomo M, Papadimitropoulos A, Cedola A, Peyrin F, Giannoni P, Pearce SG, et al. Engineering of bone using bone marrow stromal cells and a silicon-stabilized tricalcium phosphate bioceramic: evidence for a coupling between bone formation and scaffold resorption. Biomaterials 2007;28:1376-84. 